\title{
Application of Compressive Sensing to Gravitational Microlensing Experiments
}

\author{
Asmita Korde-Patel ${ }^{1,2}$, Richard K. Barry ${ }^{1}$ and Tinoosh Mohsenin ${ }^{2}$ \\ ${ }^{1}$ NASA Goddard Space Flight Center, Greenbelt, MD, USA \\ ${ }^{2}$ University of Maryland, Baltimore County, Baltimore, MD, USA \\ email: asmita.a.korde@nasa.gov
}

\begin{abstract}
Compressive Sensing is an emerging technology for data compression and simultaneous data acquisition. This is an enabling technique for significant reduction in data bandwidth, and transmission power and hence, can greatly benefit space-flight instruments. We apply this process to detect exoplanets via gravitational microlensing. We experiment with various impact parameters that describe microlensing curves to determine the effectiveness and uncertainty caused by Compressive Sensing. Finally, we describe implications for space-flight missions.
\end{abstract}

Keywords. Compressive Sensing, Gravitational Microlensing

\section{Introduction}

Gravitational microlensing is a relatively new technique that permits astronomers to characterize faint or non-emitting objects such as exoplanets or neutron stars by their effect on the apparent brightness of a distant light source as a function of time. The detailed shape of microlensing light curves provide useful science regarding the foreground lensing body or system. Hence, it is essential to capture the microlensing curve in such a way that its characteristics and shape are preserved, which typically requires high cadence observation. In order to accurately capture a microlensing curve, a good spatial image needs to be acquired. High resolution signal acquisition techniques are becoming a necessity in today's world to solve our universe's scientific mysteries. Due to data bandwidth issues for space flights, high rate sampling is not always feasible. Compressive sensing (CS) can provide a possible solution to acquiring high cadence, while attaining high resolution. CS is a new mathematical framework for sampling data at a rate lower than the Nyquist rate. CS works by exploiting sparsity in signals. In gravitational microlensing, the events tend to be sparse in spatial domain when differenced in time domain. If an image is sparse, it can be wasteful to capture all the pixels. Theoretically, CS efficiently captures signals, so that the germane information is preserved during reconstruction. In this work, we do preliminary CS research on gravitational microlensing data sets to understand the limitations and effectiveness of CS.

\section{Background}

In this section we discuss gravitational microlensing as well as compressive sensing theory.

\subsection{Gravitational Microlensing}

Gravitational microlensing is a phenomenon we observe when there is magnification in the flux of the source star due to a lensing star or planet which bends light as the source star passes through the Einstein's ring radius of the lensing star. Light from a source 
star can be observed without any perturbations if there is no concentrated mass aligned with the source star and observer. This alignment is a rare occurrence. If a star or a planet-star system is present, this body can deflect light due to its gravitational effects. This in turn, magnifies the flux of the source star we are observing. Depending on the characteristics of the system, the length of such an event can range from hours to years. Typically, they are on the order of a month (Seager, 2010). If a high enough cadence is not achieved in the detector system, a short-lived planetary perturbation can be missed. In addition, increasing the number of masses in a lensing system can significantly increase complications in the observed microlensing curves. Hence, if high resolution and high cadence are not achieved, the resolution of the curves can be greatly compromised. In order to distinguish microlensing events from other constant star flux sources, difference imaging process is applied. Difference imaging algorithm provides an output image consisting of either star sources experiencing microlensing events or cosmic events. Since the precise alignment of a source and lensing system is rare, microlensing events are rare. Post-differencing, gravitational microlensing events tend to be sparse in the spatial domain (Bramich, 2008).

\subsection{Single Lens Events}

In all the simulations, a single lens model, the simplest model is used. In a single lens event, the lensing system consists of only a lensing star. This is the simplest case we can first study. By assuming a point source, the magnification of the source star at each time is given by the source-lens separation, $\mu_{0}$, peak magnification time, $t_{0}$, and Einstein's ring radius crossing time, $t_{e}$. As $\mu_{0}$ decreases, the peak magnification increases, creating a steeper magnification curve.

\subsection{Compressive Sensing Theory}

Compressive sensing (CS) is a mathematical theory for sampling at a rate much lower than the Nyquist rate, and yet, reconstructing the signal back with little or no loss of information. CS solves for equation 2.1 to obtain a sparse solution for $x$.

$$
y=\phi x
$$

Here, $y$ is $\mathrm{Mx} 1, \phi$ is $\mathrm{MxN}$, and $x$ is $\mathrm{Nx} 1$. In CS, $\mathrm{M}<<\mathrm{N}$, hence, providing an underdetermined system to solve. Given $y$ as the obtained measurements vector and $\phi$ as the measurement matrix, we can solve for the original image, transformed into a $1 \mathrm{D}$ vector, $x$. A unique solution is obtained if the original signal is sparse in some domain. The basis of $\phi$ matrix needs to be incoherent with the sparse domain basis of the original image. As a general rule, random matrices tend to be universally incoherent and hence, are a good starting point for initial CS research. Reconstruction algorithms include various optimization algorithms as well as greedy algorithms (Eldar and Kutynoik, 2012).

\subsection{Implications}

For space flight instruments, the measurement matrix will depict a projection from the detector side. Each measurement is obtained by summing the total flux received due to each projection. This sum corresponds to one element in $y$ vector. Using very few such random projections, the number of measurements acquired will also be very less. This can significantly reduce data bandwidth and volume, which is a huge problem in space flight instruments. 

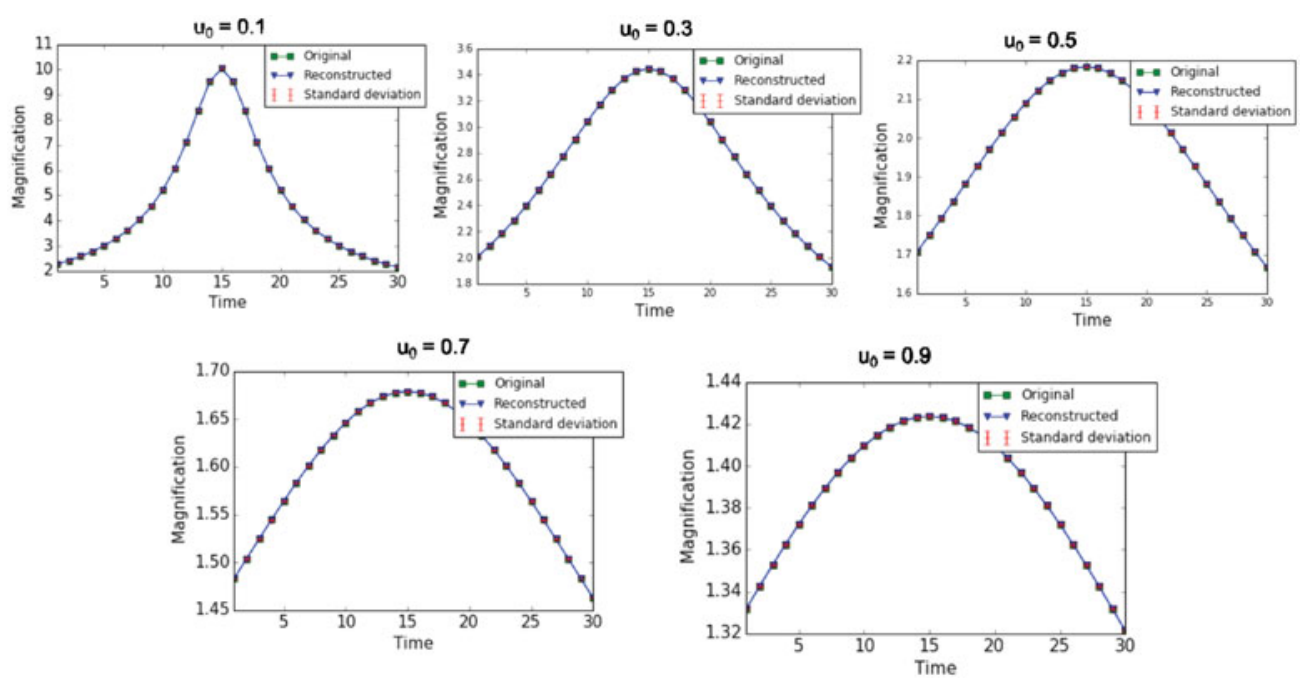

Figure 1. Original and reconstructed microlensing curves with error bars for lens-source separation parameter $\mu_{0}$ ranging from 0.1 to 0.9 .

\section{Simulations and Results}

In this research, we analyze the effects of compressive sensing on low magnification events due to large lens-source separations, normalized in terms of Einstien's ring radius. The lens-source separation parameter, $\mu_{0}$, influences the magnification of the microlensing curve. We determine the uncertainty caused as we vary $\mu_{0}$ from 0.1 units to 0.9 units of Einstein's ring radius. We use $4 \%$ of Nyquist rate measurements. In this context, Nyquist rate refers to the total number of pixels in the image, which traditional methods would need to capture.

\subsection{Simulation Setup}

We use a $25 \times 25$ pixel image, with a source star at the center of the image. The measurement matrix consists of Bernoulli random values of 0's and 1's. For each time value, 100 Monte Carlo simulations are performed by varying the measurement matrix. The center pixel in each image is portrayed as the source star with Gaussian distribution of flux in its surrounding pixels. Hence, while analyzing reconstruction of the microlensing curve, total flux or magnification is calculated using the center pixel with a 3 pixel radius. For all simulations, number of measurements, $\mathrm{M}$, is $4 \%$ of $\mathrm{N}$, where $\mathrm{N}$ is the total number of pixels in the image. Reconstruction is performed using an optimization algorithm provided in a software package by O'Donoghue and all (2016).

\subsection{Results}

Reconstructed microlensed curves for varying impact parameters are shown in Figure 1.

Compressive sensing works well for low magnification events as seen from Figure 1. However, as the magnification decreases, uncertainty increases. In order to ensure key characteristics in the microlensing curve are captured, the uncertainty at peak magnification time, $t_{0}$, needs to be less than the difference in magnitude at the time before (or after) $t_{0}$. The ratio of uncertainty at $t_{0}$ to the difference in magnification between $t_{0}$ and $t_{0}+/-1$ is shown in Table 1 . As $\mu_{0}$ reaches 0.9 units of Einstein's ring radius, the ratio reaches slightly above 1 , indicating the limits of CS reconstruction resolution. One way to overcome such a limitation is to increase the number of measurements. When we increase 
Table 1. Ratio of uncertainty at $t_{0}$ to the difference in magnification at $t_{0}$ and $t_{0}+/-1$. This is to ensure that uncertainty is within limits to acquire a good resolution at peak time

\begin{tabular}{|l|c|}
\hline$u_{0} \mid$ uncertainty at $t_{0}:$ difference in magnitude between $t_{0}$ and $t_{0}+/-1 \mid$ \\
\hline 0.1 & 0.0012 \\
0.3 & 0.0268 \\
0.5 & 0.192 \\
0.7 & 0.334 \\
0.9 & 1.046 \\
\hline
\end{tabular}

Table 2. Average \% error over all time values for each $\mu_{0}$ using $4 \%$ of $\mathrm{N}$ measurements

\begin{tabular}{|l|l|}
\hline$u_{0}$ & Average \% error \\
\hline 0.1 & 0.00160 \\
0.3 & 0.00258 \\
0.5 & 0.00272 \\
0.7 & 0.00301 \\
0.9 & 0.00334 \\
\hline
\end{tabular}

$\%$ of measurements for $\mu_{0}=0.9$ from $4 \%$ to $5 \%$, the ratio decreases to 0.84 . Table 2 shows average \% error over 100 Monte Carlo simulations, over all time values. Error is calculated using the difference in magnitude between the original and reconstructed image at the center pixel with a 3 pixel radius. From table 2 , all $\mu_{0}$ values give average reconstruction $\%$ error to be within 0.0000334 units of flux. Although \% error is relatively low, uncertainty can reach a limiting factor as the lens-source characteristic distance increases. This leads to a trade-off between optimal number of measurements and resolution uncertainty.

\section{Conclusion}

CS shows potential to reduce data in the acquisition process itself while reconstructing the signal with good accuracy, such that the characteristics of the microlensing curve are preserved. For an ideal image with only $0.1 \%$ significant (non-zero value) pixels, the microlensing curve can be reconstructed accurately using only $4 \%-5 \%$ of measurements used in traditional sampling methods. This technique could potentially be beneficial not only for low lens-source separation systems but also for separations that are much greater causing less changes in magnification over the course of a microlensing event. Miniaturized space-flight instruments which have data bandwidth, power, and volume limitations may greatly benefit from this technique.

\section{References}

Eldar, Y. \& Kutynoik, G. 2012, Compressed Sensing, Cambridge University Press

O'Donoghue, B., Chu, E., Parikh, N., \& Boyd, S. 2016, Conic Optimization via Operator Splitting and Homogenous Self-Dual Embedding, J. Optim Theory Appl, 169: 1042-1068

Seager, S. 2010, Exoplanets, The University of Arizona Press

Bramich, D. 2008, A new algorithm for difference image analysis, Monthly Notices of the Royal Astronomical Society: Letters 386.1: L77-L81 